\title{
Maoecrystal Z, a Cytotoxic Diterpene from Isodon eriocalyx with a Unique Skeleton
}

Quan-Bin Han, ${ }^{\dagger, \S}$ Susan Cheung, ${ }^{\ddagger}$ Joseph Tai, ${ }^{\ddagger}$ Chun-Feng Qiao, ${ }^{\dagger}$ Jing-Zheng Song, ${ }^{\dagger}$ Ting-Fai Tso, ${ }^{\dagger}$ Han-Dong Sun, ${ }^{\S}$ Hong-Xi Xu ${ }^{*}{ }^{\dagger}$

Chinese Medicine Laboratory, Hong Kong Jockey Club Institute of Chinese Medicine, Hong Kong, P. R. China, Departments of Pathology and Pediatrics, Center for Complementary Medicine Research, Child and Family Research Institute, University of British Columbia, Vancouver, BC, Canada, State Key Laboratory of Phytochemistry and Plant Resources in West China, Kunming Institute of Botany, Chinese Academy of Sciences, Kunming 650204, Yunnan, P. R. China xuhongxi@hkjcicm.org

Supporting information:

1D (Bruker AM-400 MHz) and 2D NMR (Bruker DRX-500 MHz) spectra of 1 in $\mathrm{C}_{5} \mathrm{D}_{5} \mathrm{~N}$
1. ${ }^{1} \mathrm{H}$ NMR
2. ${ }^{13} \mathrm{C}$ NMR
3. $\mathrm{HMQC}$
4. HMBC
5. ROESY 
1. ${ }^{1} \mathrm{H}$ NMR spectrum of compound $\mathbf{1}$

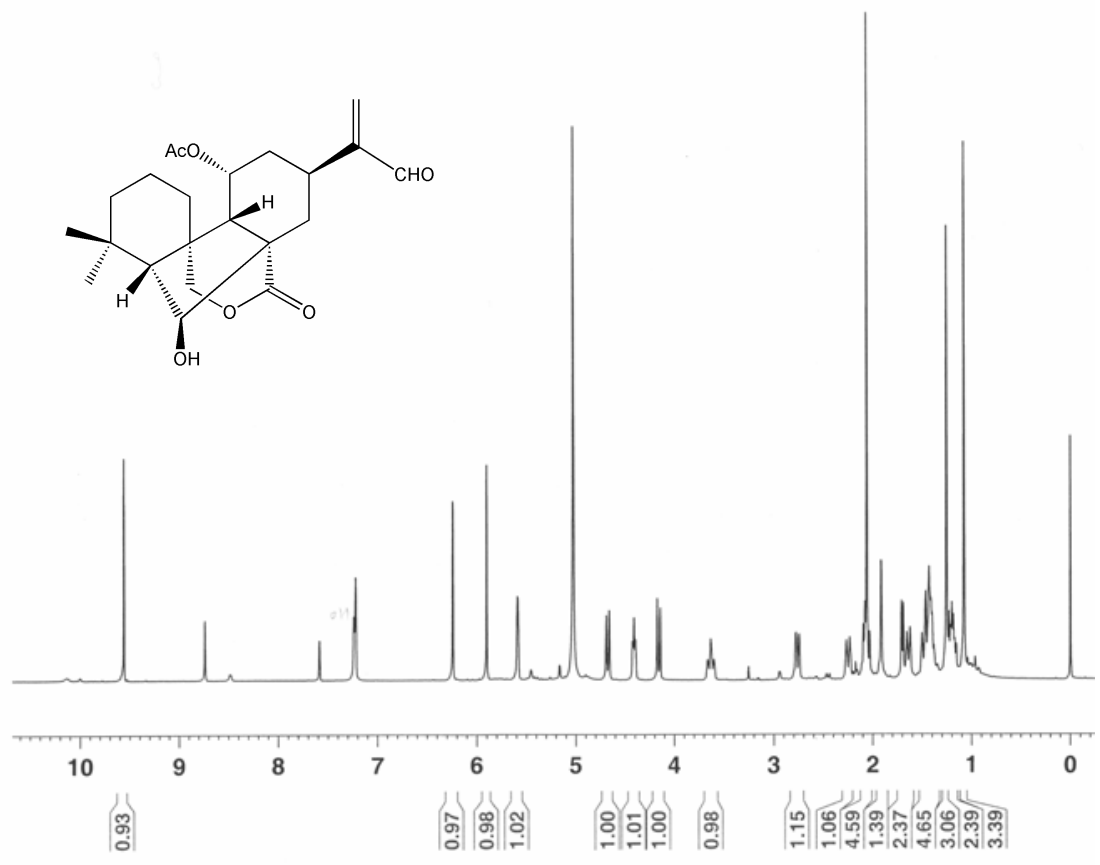

2. ${ }^{13} \mathrm{C}$ NMR spectrum of compound $\mathbf{1}$

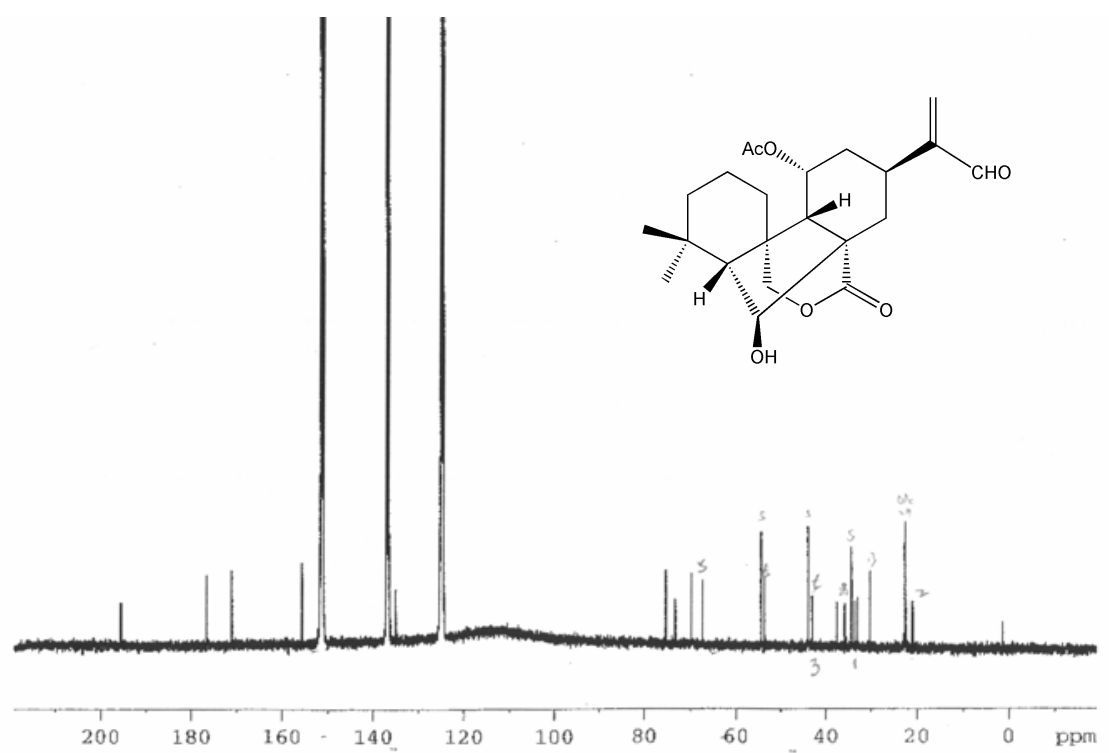


3. HMQC spectrum of compound $\mathbf{1}$

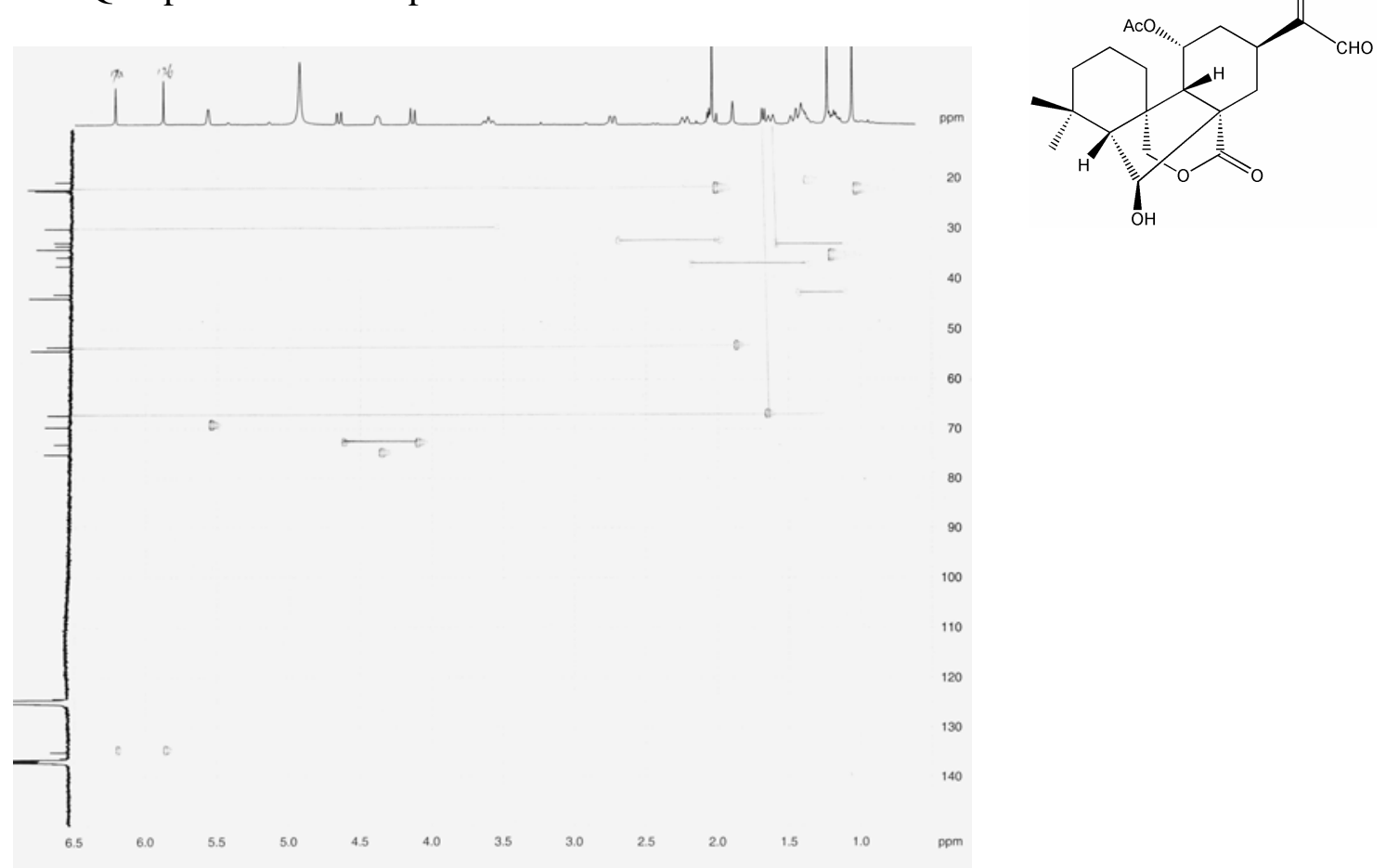

4. HMBC spectrum of compound $\mathbf{1}$

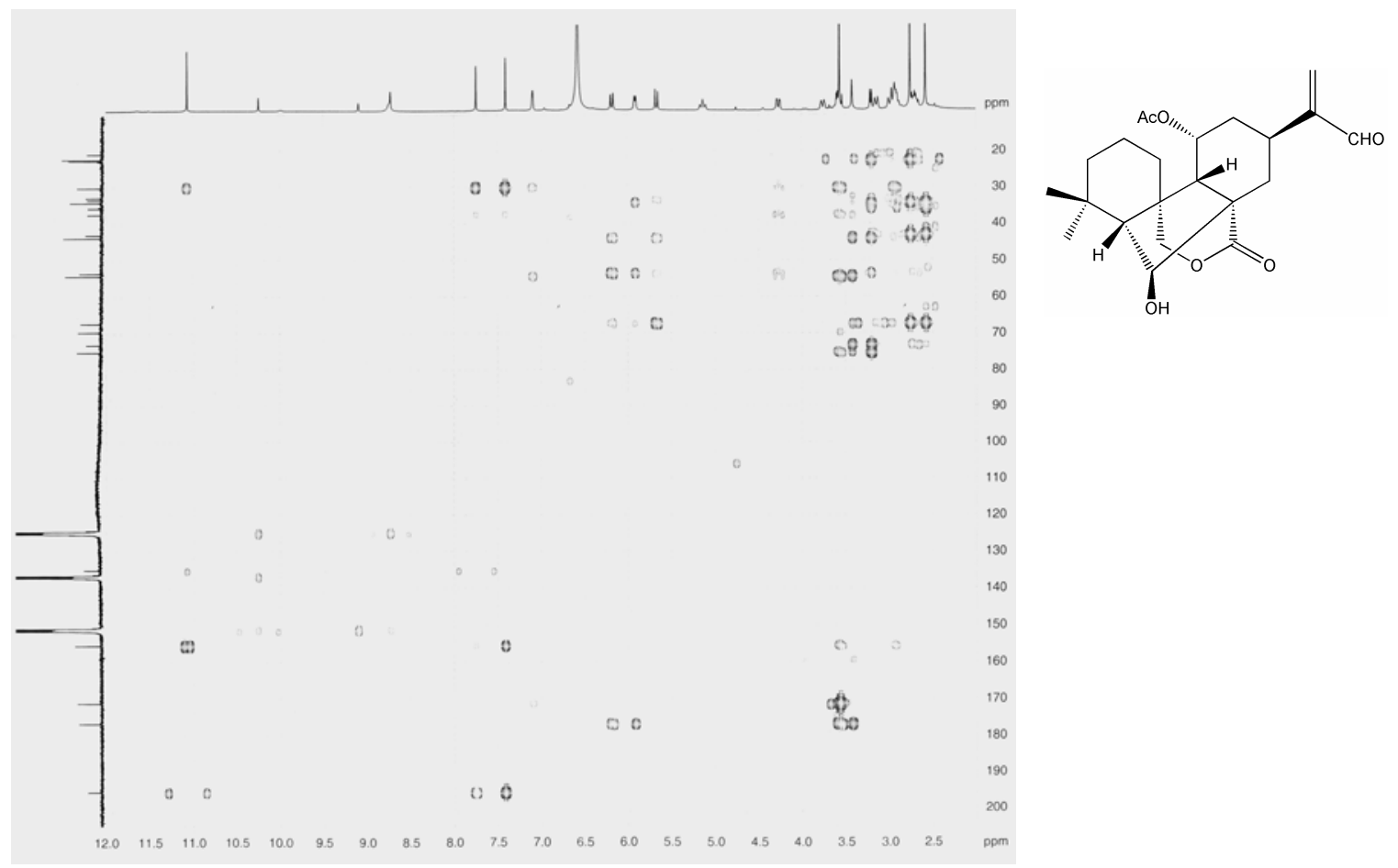




\section{ROESY spectrum of compound $\mathbf{1}$}

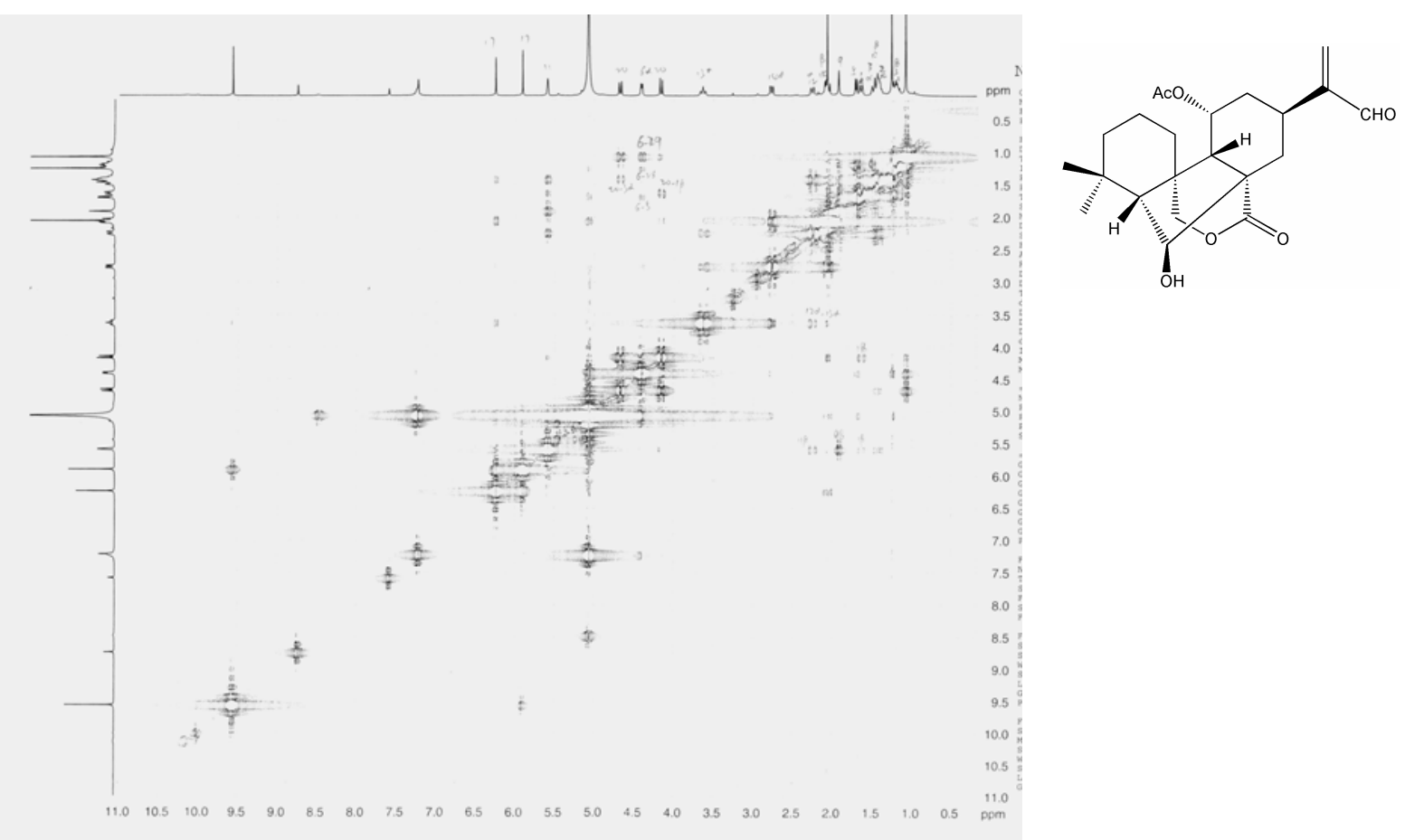

